\title{
Towards a Systematic Solution for Differential Games with Limited Communication
}

\author{
T. Mylvaganam and A. Astolfi
}

\begin{abstract}
The main aim of this work is to develop a systematic approach for dealing with differential games with limited communication. To this end a differential game with limited communication is considered. The communication topology is described by a directed graph. The main components characterising the differential game with limited communication are then identified before the resulting game is then formally defined. Sufficient conditions to solve the problem are identified both in the general nonlinear case and in the linear-quadratic case. A numerical example illustrating the theoretical approach and results is presented. Finally, several directions for further developments are identified.
\end{abstract}

\section{INTRODUCTION}

Differential game theory provides a framework to study problems in which a dynamical system is influenced by several individuals, referred to as players [1]-[3]. A wide range of problems can be described in this setting. For example, game theory and differential games have traditionally played an important role in applications related to military and defense, and economics [4], [5]. Differential games play a role, either as an analysis or design tool, in a variety of other areas, such as biological systems [6]-[9], power systems [10][13] and multi-agent systems [14], [15] to mention just a few. Moreover, for the special case in which there is only one player, a differential game boils down to a standard optimal control problem, see for example [16].

Unlike optimal control problems, different solution concepts exist when studying differential game problems: two notable examples are Nash and Stackelberg equilibrium solutions [2], [3], [17]. The most common solution concept is the Nash equilibrium solution [1][3], [18]-[20]. Within the context of Nash equilibrium solutions it is assumed that all players are rational, are aware of one another's performance criteria and that all players announce their strategies simultaneously [1], [2]. Although this solution concept describes a wide range of scenarios, in many practical applications the

This work has been conducted as part of the research project "Stability and Control of Power Networks with Energy Storage (STABLE-NET)" project which is funded by the RCUK's Energy Programme (contract no: EP/L014343/1). T. Mylvaganam is with the Department of Electrical and Electronic Engineering, Imperial College London, London SW7 2AZ, UK (Email:thulasi.mylvaganam06@imperial.ac.uk).

A. Astolfi is with the Department of Electrical and Electronic Engineering, Imperial College London, London SW7 2AZ, UK and the DICII, Università di Roma "Tor Vergata", Via del Politecnico 1, 00133 Roma, Italy underlying assumption on the required exchange of information is difficult to satisfy. For example, a player may be ignorant about the existence of some or all of the other players or the communication between players may be limited. This becomes important in certain engineering applications in which communication may be expensive or unavailable. In such settings centralised control schemes cannot be adopted and it is necessary to develop distributed control strategies instead [21]. With this motivation it is of interest to adapt the framework provided by differential game theory to deal with problems in which the communication between the players is limited.

In the literature, differential games in which the players have limited sensing abilities, i.e. the case in which the players do not have full information regarding the state of the system, have been studied for different applications. For example, in [22], [23] pursuit-evasion games with limited sensing ranges are considered. However, there is still a need to explore and formalise problems in which the communication between players is limited. Stackelberg solutions for differential games allow to study problems in which there is a certain hierarchy between the players, i.e. the players announce their strategies in a certain order [19], [24]. However, in many applications the communication between players may be even more restrictive than this. It is therefore of interest to consider differential games with diverse communication structures.

In this paper we consider differential games in which the communication between agents is described by a time-invariant, directed graph in which nodes represent players and edges represent the existence of a communication link between two players. This graph theoretical approach is similar to what is commonly done when dealing with multi-agent systems with limited communications [25].

The aim of this work is to develop a framework to formally define and study problems with limited information exchange between the players in a general setting. To do this we identify which information is exchanged between communicating players and which assumptions are made regarding players which do not share a communication link. We are now in a position to define the problem and move towards developing a framework for systematically dealing with differential games with limited communication.

The remainder of the paper is structured as follows. 
In Section II the differential game with limited communication is introduced: the term "limited communications" is explained in detail before the problem formulation is defined. In Section III sufficient conditions to solve the differential game are presented both in the nonlinear case and in the linear-quadratic case. A numerical example involving a multi-agent system is presented to illustrate the results in Section IV. For this particular example, it is shown that, in addition to accommodating for limited communication between the agents, the solution is such that the agents (players) do not need full access to the state. Some concluding remarks and directions for future research are given in Section V.

\section{Problem Formulation}

Consider a scenario in which $N>0$ players influence a dynamical systems via the selection of their control inputs $u_{i}(t) \in \mathbb{R}^{m_{i}}, i=1, \ldots, N$, more commonly referred to as control strategies within the game theoretical framework. The players are denoted by subscripts $1, \ldots, N$. Moreover, consider the case in which the system is input-affine with the dynamics

$$
\dot{x}=f(x)+g_{1}(x) u_{1}+\ldots, g_{N}(x) u_{n},
$$

where $x(t) \in \mathbb{R}^{n}$, with $n>0$, is the state of the system, $f(x): \mathbb{R}^{n} \rightarrow \mathbb{R}^{n}$ and $g_{i}(x) \in \mathbb{R}^{n \times m_{i}}$ with $m_{i}>0$, for $i=1, \ldots, N$, are smooth mappings.

We assume that the origin is an equilibrium of the system, i.e. $f(0)=0$.

Each player selects its control strategy to optimise its own performance criterion which may, or may not, be conflicting with the performance criteria of one or more of the other players. The objective of a player is quantified by a cost functional which it seeks to minimise. In this paper we focus on feedback equilibrium strategies, i.e. $u_{i}(t)=u_{i}(x(t))$. When considering standard feedback Nash equilibrium solutions it is assumed that all players have information regarding the cost functionals associated with, and strategies adopted by all the other players. Instead, we consider the problem in which the players only communicate information regarding their objectives and strategies to a set of so-called neighbouring agents. We consider the case in which the communication topology is fixed, i.e. it is time invariant.

In this case the communication between the players can be represented by a time-invariant, directed graph consisting of $N$ nodes, labelled $i, \ldots, N$, and a set of edges: each node represents a player and each edge represents a communication link between two players. If player $i$ communicates with player $j$ a directed edge exists from node $i$ to node $j$ and player $j$ is said to be a neighbour of player $i$. Note that the communication is not necessarily bidirectional, i.e. player $j$ being a neighbour of player $i$ does not imply that player $i$ is a neighbour of player $j$. Let
$\mathcal{N}_{i}$ denote the set of players which are neighbours of player $i .{ }^{1}$ Let $\mathcal{G}$ denote the graph describing the communication topology. In Figure 1 an example of a graph describing the communication topology between a set of 5 players is depicted. The arrows represent the edges and the "direction" of the communication, e.g. the arrow connecting node 3 and node 1 indicates that player 3 shares its information with player 1 . In this case, for example, $\mathcal{N}_{1}=\{2,3,5\}$ and $\mathcal{N}_{2}=$ $\{4\}$. Approaches based on graph theory are frequently used to describe the communication between agents in multi-agent systems as seen, for instance, in [25] and references therein, where also a background on graph theory is provided. For more examples on graph theoretic methods and their applications to multi-agent systems see, for example, [26], [27].

Let $\mathbf{u}_{\mathbf{j} \in \mathcal{N}_{\mathbf{i}}}$ denote the set of strategies of the neighbours of the $i$-th player, i.e. $\mathbf{u}_{\mathbf{j} \in \mathcal{N}_{\mathbf{i}}}=\left\{u_{j}: j \in \mathcal{N}_{i}\right\}$. Given a communication topology, the cost functional ${ }^{2}$ of each player is given by

$$
\begin{aligned}
J_{i}\left(x(0), u_{i}, \mathbf{u}_{\mathbf{j} \in \mathcal{N}_{\mathbf{i}}}\right) \triangleq & \frac{1}{2} \int_{0}^{\infty}\left(q_{i}(x(t))+\alpha_{i} u_{i}(t)^{\top} u_{i}(t)\right. \\
& \left.+\sum_{j \in \mathcal{N}_{i}} \alpha_{i j} u_{j}(t)^{\top} u_{j}(t)\right) \mathrm{d} t,
\end{aligned}
$$

where $\alpha_{i}>0$ and $\alpha_{i j}$ are constant parameters, and $q_{i}(x) \geq 0$ is a running cost satisfying $q_{i}(0)=0$, for $j \in \mathcal{N}_{i}$ and $i=1, \ldots, N$.

In what follows we assume that the following statement holds true

Assumption 1: The running costs in (2), $i=1, \ldots, N$ are such that

$$
\sum_{i=1}^{N} q_{i}(x)>0,
$$

for all $x \neq 0$

Remark 1: If the communication links are not identical, for example if some players communicate more easily with certain other players, a weighted graph can be used to represent the communication topology.

Since the players do not have any information regarding the objectives or strategies of nonneighbouring players, each player must make certain assumptions regarding the strategies of its nonneighbouring players. Suppose that the $i$-th player assumes that $u_{j}(t)=\bar{u}_{i j}$ for all $j=1, \ldots, N$ and $j \notin \mathcal{N}_{i}$. That is, if the $j$-th player is not a neighbour of the $i$-th player, the $i$-th player assumes that the strategy of the $j$-th player is a certain function of the state $x$, which we denote by $\bar{u}_{i j}(x)$. Let $\overline{\mathbf{u}}_{\mathbf{j} \notin \mathcal{N}_{\mathbf{i}}}$ denote the assumptions

\footnotetext{
${ }^{1} \mathcal{N}_{i}$ is said to be the neighbouring set of player $i$ and in this definition of the set, a player is not said to be a neighbour of itself. Note that alternative definitions exist.

${ }^{2}$ Note that the strategies of players which are not neighbours of player $i$ cannot appear in the cost functional associated with player $i$ since this information is only shared by neighbouring players.
} 


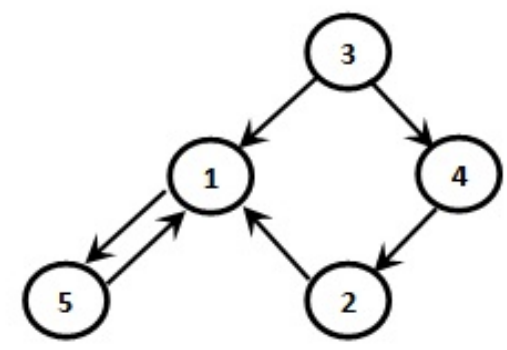

Fig. 1. Example of graph representing communication between a set of 5 players

the $i$-th player makes regarding the strategies of nonneighbouring players, i.e. $\overline{\mathbf{u}}_{\mathbf{j} \notin \mathcal{N}_{\mathbf{i}}}=\left\{\bar{u}_{i j}(x): j \notin \mathcal{N}_{i}\right\}$.

Remark 2: In some settings, for example in a stock market or a distributed power grid, a player may not be aware of the existence of all players. For example, it is possible that the $i$-th player is aware of and shares information with its neighbours only, whereas it is unaware about the existence of the other players. This setting could be described by the selection $\bar{u}_{i j}(x)=0$ for all $x$ and for all $j \notin \mathcal{N}_{i}$.

Using this setup the differential game with limited communication can be defined as follows.

Problem 1: Consider the $N$-player differential game characterised by the dynamical system (1), the cost functionals (2), $i=1, \ldots, N$, the graph $\mathcal{G}$ and the assumptions $\bar{u}_{i j}$ for $j \notin \mathcal{N}_{i}$ and $i=1, \ldots, N$. The problem of solving the differential game with limited communication lies in determining a set of feedback strategies $\mathbf{u}^{*}=\left\{u_{1}^{*}, \ldots, u_{N}^{*}\right\}$ which is such that the origin of the system (1) in closed-loop with $\mathbf{u}^{*}$ is (locally) asymptotically stable. Furthermore, $\mathbf{u}^{*}$ must satisfy the inequalities

$$
J_{i}\left(x(0), u_{i}^{*}, \mathbf{u}_{\mathbf{j} \in \mathcal{N}_{\mathbf{i}}}^{*}, \overline{\mathbf{u}}_{\mathbf{j} \notin \mathcal{N}_{\mathbf{i}}}\right) \leq J_{i}\left(x(0), u_{i}, \mathbf{u}_{\mathbf{j} \in \mathcal{N}_{\mathbf{i}}}^{*}, \overline{\mathbf{u}}_{\mathbf{j} \notin \mathcal{N}_{\mathbf{i}}}\right)
$$

for all $u_{i} \neq u_{i}^{*}$ such that the the origin of the system (1) in closed-loop with the set of strategies $\left\{u_{i}, \mathbf{u}_{\mathbf{j} \in \mathcal{N}_{\mathbf{i}}}^{*}, \mathbf{u}_{\mathbf{j} \in \mathcal{N}_{\mathbf{i}}}^{*}\right\}$ is (locally) asymptotically stable, for $i=1, \ldots, N$.

Remark 3: The inequalities (3) can be interpreted as follows. If the $i$-th player assumes $u_{j}=u_{j}^{*}$ for all $j \in \mathcal{N}_{i}$ and $u_{j}=\bar{u}_{i j}$ for all $j \notin \mathcal{N}_{i}$, it cannot perform any better by deviating from the strategy $u_{i}^{*}$. The idea behind this solution is similar to that of Nash equilibrium solutions, but is here recast in a distributed setting.

Note that whereas a standard differential game (when interested in Nash equilibirum solutions) is characterised by the dynamics (1) and the cost functionals (2), for $i=1, \ldots, N$, the differential game with limited communication is characterised by the graph $\mathcal{G}$ describing the communication topology and the set of assumptions $\bar{u}_{i j}(x)$, for all $j \notin \mathcal{N}_{i}$ and $i=1, \ldots, N$, in addition to the dynamics (1) and the cost functionals (2), for $i=1, \ldots, N$.

\section{SOLUTION TO THE DIFFERENTIAL GAME WITH LIMITED COMMUNICATION}

Using dynamic programming and the principle of optimality sufficient conditions for a solution of Problem 1 to exist are given in this section [1], [28]. The general nonlinear setting is described before the special case of linear-quadratic differential games is considered. For simplicity we consider first the case in which $\alpha_{i j} \geq 0$ in (2), for $j \in \mathcal{N}_{i}$ and $i=1, \ldots, N$. However, the more general case can be considered using the same arguments as will be remarked at the end of this section.

\section{A. Nonlinear setting}

Consider Problem 1 and the system of partial differential equations (PDEs)

$$
\begin{gathered}
\frac{1}{2} q_{i}(x)-\frac{1}{2 \alpha_{i}} \frac{\partial V_{i}}{\partial x} g_{i}(x) g_{i}(x)^{\top} \frac{\partial V_{i}}{\partial x}+\frac{\partial V_{i}}{\partial x} f(x) \\
+\sum_{j \in \mathcal{N}_{i}} \frac{1}{\alpha_{j}} \frac{\partial V_{i}}{\partial x} g_{j}(x) g_{j}(x)^{\top} \frac{\partial V_{j}}{\partial x}+\sum_{j \notin \mathcal{N}_{i}} \frac{\partial V_{i}}{\partial x} g_{j}(x) \bar{u}_{i j}(x) \\
+\frac{1}{2} \sum_{j \in \mathcal{N}_{i}} \frac{\alpha_{i j}}{\alpha_{j}^{2}} \frac{\partial V_{j}}{\partial x} g_{j}(x) \top g_{j}(x)^{\top} \frac{\partial V_{j}}{\partial x}=0,
\end{gathered}
$$

where $V_{i}(x): \mathbb{R}^{n} \rightarrow \mathbb{R}$ and $V_{i}(0)=0$, for $i=1, \ldots, N$.

Theorem 1: Suppose we can find a solution to the coupled PDEs (4), $i=1, \ldots N$, and suppose the solution is such that $\sum_{i=1}^{N} V_{i}(x)>0$ for all $x \neq 0$ and $V_{i}(0)=0$ holds for all $i=1, \ldots, N$. Furthermore, suppose that the solution is such that the condition

$$
\begin{aligned}
& \frac{1}{2} \sum_{i=1}^{N}\left(q_{i}(x)+\frac{1}{\alpha_{i}} \frac{\partial V_{i}}{\partial x} g_{i}(x) g_{i}(x)^{\top} \frac{\partial V_{i}^{\top}}{\partial x}\right) \\
& +\frac{1}{2} \sum_{i=1}^{N} \sum_{j \in \mathcal{N}_{i}} \frac{\alpha_{i j}}{\alpha_{j}^{2}} \frac{\partial V_{j}}{\partial x} g_{j}(x) g_{j}(x)^{\top} \frac{\partial V_{j}^{\top}}{\partial x}> \\
& \sum_{i=1}^{N} \sum_{j \notin \mathcal{N}_{i}} \frac{1}{\alpha_{j}} \frac{\partial V_{i}}{\partial x} g_{j}(x)\left(g_{j}(x)^{\top} \frac{\partial V_{j}^{\top}}{\partial x}-\alpha_{j} \bar{u}_{i j}(x)\right),
\end{aligned}
$$

holds for all $x \neq 0$. Then the set of feedback strategies given by

$$
u_{i}^{*}=-\frac{1}{\alpha_{i}} g_{i}(x)^{\top} \frac{\partial V_{i}^{\top}}{\partial x},
$$

for $i=1, \ldots, N$, is a solution to Problem 1 .

Proof: The proof consists of two steps. Firstly it is shown that the set of strategies (6), $i=1, \ldots, N$, 
are such that the inequalities (3) are satisfied for $i=$ $1, \ldots, N$. Secondly it is shown that the zero equilibrium of the closed-loop system is (locally) asymptotically stable.

Each player can be associated with its Hamiltonian function

$$
\begin{aligned}
& H_{i}\left(x, \lambda_{i}, u_{i}, \mathbf{u}_{\mathbf{j} \in \mathcal{N}_{\mathbf{i}}}, \overline{\mathbf{u}}_{\mathbf{j} \notin \mathcal{N}_{\mathbf{i}}}\right) \\
& \quad=\frac{1}{2} q_{i}(x)+\frac{1}{2} \alpha_{i} u_{i}^{\top} u_{i}+\frac{1}{2} \sum_{j \in \mathcal{N}_{i}} \alpha_{i j} u_{j}^{\top} u_{j} \\
& \quad+\lambda_{i}^{\top}\left(f(x)+g_{i}(x) u_{i}+\sum_{j \in \mathcal{N}_{i}} g_{j}(x) u_{j}+\sum_{j \notin \mathcal{N}_{i}} g_{j}(x) \bar{u}_{i j}\right),
\end{aligned}
$$

where $\lambda_{i}$ is the costate. The feedback strategy $u_{i}=$ $-\frac{1}{\alpha_{i}} g_{i}(x)^{\top} \lambda_{i}$ minimises the Hamiltonian associated with the $i$-th player. It follows from the dynamic programming principle that if a solution to the PDEs (4), $i=1, \ldots, N$, can be found the set of strategies (6), $i=1, \ldots, N$ satisfies the inequalities (3).

It now remains to show that the origin is an equilibrium of the closed-loop system (1)-(6). Let $W(x)=$ $\sum_{i=1}^{N} V_{i}(x)$ be a candidate Lyapunov function and note that $W(x)>0$ for all $x \neq 0$. The time derivative of the function is given by

$$
\begin{aligned}
\dot{W}= & \sum_{i=1}^{N} \frac{\partial V_{i}}{\partial x}\left(f(x)+\sum_{i=1}^{N} g_{i}(x) u_{i}^{*}\right) \\
= & -\frac{1}{2} \sum_{i=1}^{N}\left(q_{i}(x)+\frac{1}{\alpha_{i}} \frac{\partial V_{i}}{\partial x} g_{i}(x) g_{i}(x)^{\top} \frac{\partial V_{i}^{\top}}{\partial x}\right) \\
& -\frac{1}{2} \sum_{i=1}^{N} \sum_{j \in \mathcal{N}_{i}} \frac{\alpha_{i j}}{\alpha_{j}^{2}} \frac{\partial V_{j}}{\partial x} g_{j}(x) g_{j}(x)^{\top} \frac{\partial V_{j}^{\top}}{\partial x} \\
& +\sum_{i=1}^{N} \sum_{j \notin \mathcal{N}_{i}} \frac{1}{\alpha_{j}} \frac{\partial V_{j}}{\partial x} g_{j}(x) g_{j}(x)^{\top}{\frac{\partial V_{j}}{\partial x}}^{\top} .
\end{aligned}
$$

It follows from (5) that $\dot{W}(x)<0$ for all $x \neq 0$. Closedloop stability of the origin follows from standard Lyapunov arguments.

Remark 4: The coupled PDEs (4), $i=1, \ldots, N$, resemble the Hamilton-Jacobi-Isaacs PDEs which are encountered in standard differential games [1], [2], [20]. However, the PDEs (4), $i=1, \ldots N$, contain terms which rely on the assumptions made by the $i$-th player regarding the strategies of non-neighbouring players, i.e. they rely on $\bar{u}_{i j}$ for $j \notin \mathcal{N}_{i}$, instead of the actual strategies adopted by the non-neighbouring players, as is the case with the standard Hamilton-Jacobi-Isaacs PDEs.
Remark 5: The inequality (5) provides a sufficient condition for $\dot{W}<0$ for all $x \neq 0$. Note that for the case in which there is full communications between all players,

$$
\bar{u}_{i j}=u_{j}^{*}=-\frac{1}{\alpha_{j}} g_{j}(x)^{\top} \frac{\partial V_{j}^{\top}}{\partial x},
$$

for $j \notin \mathcal{N}_{i}$ and $i=1, \ldots, N$. In this case the right-handside of the inequality (5) is zero and the inequality is trivially satisfied by Assumption 1 .

\section{B. Linear-Quadratic setting}

Consider the case in which the dynamics (1) is linear, i.e.

$$
\dot{x}=A x+\sum_{i=1}^{N} B_{i} u_{i},
$$

where $A \in \mathbb{R}^{n \times n}$ and $B_{i} \in \mathbb{R}^{n \times m_{i}}, i=1, \ldots, N$, are constant matrices, whereas the running costs are quadratic, i.e. they are of the form $q_{i}(x)=x \top Q_{i} x$, where $Q_{i} \in \mathbb{R}^{n \times n}$ and $Q_{i} \geq 0, i=1, \ldots, N$. In optimal control and standard differential games this linearquadratic setting leads to the linear regulator problem and linear-quadratic differential games, respectively. Both problems have been extensively studied in the literature: see, for example, [16], [29]. The reasons for considering this special case are twofold. Firstly, as it is seen with linear regulator problems and standard linear-quadratic differential games, the PDEs (4) can be replaced by algebraic Riccati equations (AREs). Secondly, since these matrix equations are sometimes ${ }^{3}$ more readily solved than the PDEs (4) this special case can help provide a better understanding of the condition (5) of Theorem 1.

As is commonly done when considering standard linear-quadratic differential games, see for example [29], [30], we limit our attention to linear feedback strategies. Thus, suppose $\bar{u}_{i j}(x)=\bar{U}_{i j} x$, where $\bar{U}_{i j} \in$ $\mathbb{R}^{n \times m_{j}}$, for $i=1, \ldots, N, j \notin \mathcal{N}_{i}$ and $j=1, \ldots, N$. The PDEs (4), $i=1, \ldots, N$ can then be replaced by the algebraic Riccati-like equations

$$
\begin{aligned}
& P_{i} A+A^{\top} P_{i}+Q_{i}-\frac{1}{\alpha_{i}} P_{i} B_{i} B_{i}^{\top} P_{i}+\sum_{j \in \mathcal{N}_{i}} \frac{\alpha_{i j}}{\alpha_{j}^{2}} P_{j} B_{j} B_{j}^{\top} P_{j} \\
& -\sum_{j \in \mathcal{N}_{i}} \frac{1}{\alpha_{j}} P_{i} B_{j} B_{j}^{\top} P_{j}-\sum_{j \in \mathcal{N}_{i}} \frac{1}{\alpha_{j}} P_{j} B_{j} B_{j}^{\top} P_{i} \\
& +\sum_{j \notin \mathcal{N}_{i}}\left(P_{i} B_{j} \bar{U}_{i j}+\bar{U}_{i j}^{\top} B_{j}^{\top} P_{i}\right)=0 .
\end{aligned}
$$

In this special case Theorem 1 reduces the following statement.

${ }^{3}$ For the case in which $N>1$ solving the coupled algebraic Riccati equations characterising a linear-quadratic differential game is not trivial and has been given attention in the literature for differential games with full communication. See, for example, [29]. 
Proposition 1: Suppose we can find a solution to the AREs such that $\sum_{i=1}^{N} P_{i}>0$ and

$$
\begin{aligned}
& \sum_{i=1}^{N}\left(Q_{i}+\frac{1}{\alpha_{i}} P_{i} B_{i} B_{i}^{\top} P_{i}+\sum_{j \in \mathcal{N}_{i}} \frac{\alpha_{i j}}{\alpha_{j}^{2}} P_{j} B_{j} B_{j}^{\top} P_{j}\right) \\
& >\sum_{i=1}^{N} \sum_{j \notin \mathcal{N}_{i}} \frac{1}{\alpha_{j}} P_{i} B_{j}\left(B_{j}^{\top} P_{j}-\bar{U}_{i j}\right) \\
& \quad+\sum_{i=1}^{N} \sum_{j \notin \mathcal{N}_{i}} \frac{1}{\alpha_{j}}\left(P_{j} B_{j}-\bar{U}_{i j}^{\top}\right) B_{j}^{\top} P_{i} .
\end{aligned}
$$

Then the set of linear feedback strategies

$$
u_{i}^{*}=-\frac{1}{\alpha_{j}} B_{i}^{\top} P_{i} x,
$$

$i=1, \ldots, N$, solves Problem 1 .

Proof: The proof follows directly from Theorem 1 with $V_{i}=\frac{1}{2} x^{\top} P_{i} x$, for $i=1, \ldots N$.

Remark 6: If $\bar{U}_{i j}=0$ for $i=1, \ldots, N, j \notin \mathcal{N}_{i}$ and $j=1, \ldots, N,(8), i=1, \ldots, N$ are standard algebraic Riccati-type equations. In Section IV a problem involving this scenario is considered.

Remark 7: It is possible to consider the case in which $\alpha_{i j}<0$ for some $j \in \mathcal{N}_{i}, i=1, \ldots, N$. However, in this case some additional care must be taken to ensure that $W(x)>0$ for all $x \neq 0$.

\section{A Numerical ExAmple}

To illustrate the theoretical results in Section III a numerical example is presented. In particular a linearquadratic problem which can be interpreted in a multiagent setting is considered.

Consider the case in which $N=3$. Each player is an agent described by its position $p_{i}(t) \in \mathbb{R}^{2}$. Suppose the players satisfy single-integrator dynamics, namely $\dot{p}_{i}=u_{i}$, where $u_{i}(t) \in \mathbb{R}^{2}$ is the control strategy of the $i$-th player. Suppose the first player seeks to reach a target $p_{1}^{*} \in \mathbb{R}^{2}$ whereas the remaining two players seek to reach certain distances relative to the first player. The relative distances are defined by the vectors $r_{12}$ and $r_{13}$ for second and third agents, respectively. Suppose the players share information regarding their objectives and strategies according to the (directed) graph shown in Figure 2 and, furthermore, suppose $\alpha_{i}=1, i=1,2,3$ and $\alpha_{i j}=0$ for $i=1, \ldots, N, j \in \mathcal{N}_{i}$ and $j=1, \ldots, N$.

This scenario is similar in spirit to the problems considered in [15], albeit here with limited communication. Defining the state as $x=$ $\left(\left(p_{1}-p_{1}^{*}\right)^{\top},\left(\left(p_{2}-p_{1}\right)-r_{12}\right)^{\top},\left(\left(p_{3}-p_{1}\right)-r_{13}\right)^{\top}\right)^{\top}$ the problem can be formulated as the differential game with limited communication characterised by the state dynamics

$$
\dot{x}=B_{1} u_{1}+B_{2} u_{2}+B_{3} u_{3},
$$

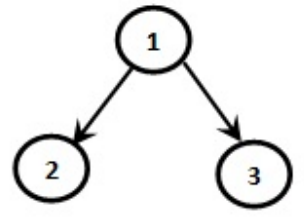

Fig. 2. Graph representing the communication topology in the 3player example.

with $^{4} B_{1}=[I,-I,-I], B_{2}=[0, I, 0]$ and $B_{3}=[0,0, I]$, the cost functionals (2) with running costs $q_{i}(x)=$ $x^{\top} Q_{i} x$, for $i=1, \ldots, N$, and the graph shown in Figure 2. Finally it is assumed that $\bar{u}_{i j}(x)=0$ for all $x$ and for all $j \notin \mathcal{N}_{i}, j=1,2,3$ and $j \neq i$, for $i=1,2,3$. Thus the problem can be described as the linear-quadratic differential game considered in Section III-B.

Let $x=\left(x_{1}^{\top}, x_{2}^{\top}, x_{3}^{\top}\right)^{\top}$ and consider the case in which

$$
\begin{gathered}
Q_{1}=\left[\begin{array}{ccc}
4 I & \mathbf{0} & \mathbf{0} \\
\mathbf{0} & \mathbf{0} & \mathbf{0} \\
\mathbf{0} & \mathbf{0} & \mathbf{0}
\end{array}\right], \quad Q_{2}=\left[\begin{array}{ccc}
I & \mathbf{0} & \mathbf{0} \\
\mathbf{0} & 2 I & \mathbf{0} \\
\mathbf{0} & \mathbf{0} & \mathbf{0}
\end{array}\right], \\
Q_{3}=\left[\begin{array}{ccc}
I & \mathbf{0} & \mathbf{0} \\
\mathbf{0} & \mathbf{0} & \mathbf{0} \\
\mathbf{0} & \mathbf{0} & 4 I
\end{array}\right] .
\end{gathered}
$$

These running costs can be interpreted as follows. Whereas the first player is only interested in reaching the target $p_{1}^{*}$, the second and third player are interested in reaching the desired positions relative the the first player as well as being interested in the first player reaching the target ${ }^{5}$. This could, for example, be understood in the context of the movement of a formation in which a leader seeks to reach a target for the group, whereas the followers seek to reach the target for the group while reaching and maintaining certain positions relative to the leader.

It follows that the matrices

$$
\begin{gathered}
P_{1}=\left[\begin{array}{ccc}
2 I & \mathbf{0} & \mathbf{0} \\
\mathbf{0} & \mathbf{0} & \mathbf{0} \\
\mathbf{0} & \mathbf{0} & \mathbf{0}
\end{array}\right], \quad P_{2}=\left[\begin{array}{ccc}
I & I & \mathbf{0} \\
I & 2 I & \mathbf{0} \\
\mathbf{0} & \mathbf{0} & \mathbf{0}
\end{array}\right], \\
P_{3}=\left[\begin{array}{ccc}
I & \mathbf{0} & I \\
\mathbf{0} & \mathbf{0} & \mathbf{0} \\
I & \mathbf{0} & 2 I
\end{array}\right],
\end{gathered}
$$

satisfy the coupled AREs (8), $i=1,2,3$. Note that it can easily be seen that the matrices are such that the conditions (9) are satisfied for all $i=1,2,3$. The linear feedback strategies (10) satisfying (3), for $i=1,2,3$, are

\footnotetext{
${ }^{4}$ The identity and zero matrices are denoted by $I$ and $\mathbf{0}$, respectively.

${ }^{5}$ Collisions are not taken into consideration in this example.
} 

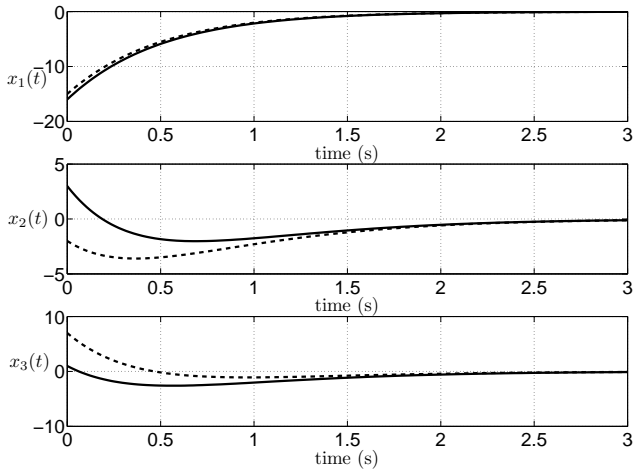

Fig. 3. Time histories of the first (solid lines) and second (dashed lines) of $x_{1}$ (top), $x_{2}$ (middle) and $x_{3}$ (bottom) corresponding to the first scenario.

then given by

$$
\begin{aligned}
& u_{1}=-2 x_{1}^{\top}, \\
& u_{2}=-\left(x_{1}^{\top}+2 x_{2}^{\top}\right), \\
& u_{3}=-\left(x_{1}^{\top}+2 x_{3}\right) .
\end{aligned}
$$

Remark 8: Due to the structure of problem, i.e. the structure of $B_{i}$ and $Q_{i}$ (and hence also of $P_{i}$ ) for $i=$ $1,2,3$, in addition to not communicating information regarding their objectives and strategies the players do not require full information regarding the state $x$. In particular, as seen in (11) the second player does not require knowledge of the position of the third player and vice versa.

Simulations of the closed-loop system (1)-(10), $i=$ $1,2,3$, have been run for two scenarios, the results of which are presented in what follows.

Consider first the scenario in which $p_{1}(0)=$ $(-6,-5)^{\top}, p_{2}(0)=(-5,-7)^{\top}$ and $p_{3}(0)=(-5,0)^{\top}$, and the desired final positions are determined by the vectors $p_{1}^{*}=(10,10)^{\top}, r_{12}=(-2,0)^{\top}$ and $r_{13}=$ $(0,-2)^{\top}$. The time histories of the first (solid lines) and second (dashed lines) of the states $x_{1}$ (top), $x_{2}$ (middle) and $x_{3}$ (bottom) are shown in Figure 3. Note that the states converge to $x=0$ as expected. The trajectories of the players on the $x, y$-plane, i.e. $p_{1}$ (solid line), $p_{2}$ (dashed line) and $p_{3}$ (dotted line) are shown in Figure 4. The square and circular markers indicate the initial and final positions of the players, respectively, whereas the arrows indicate the direction of travel.

Consider now a second scenario in which $p_{1}(0)=$ $(-1,1)^{\top}, p_{2}(0)=(-5,5)^{\top}$ and $p_{3}(0)=(-5,-5)^{\top}$, whereas $p_{1}^{*}, r_{12}$ and $r_{13}$ are as in the previous scenario. The time histories of the first (solid lines) and second (dashed lines) of the states $x_{1}$ (top), $x_{2}$ (middle) and $x_{3}$ (bottom) are shown in Figure 5. The trajectories of the three players are shown in Figure 6 where the solid, dashed and dotted lines indicate the first, second

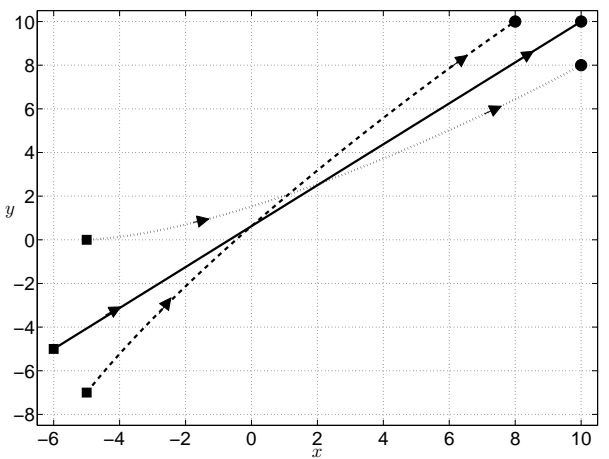

Fig. 4. Time trajectories of $p_{1}$ (solid line), $p_{2}$ (dashed line) and $p_{3}$ (dotted line) on the $x, y$-plane corresponding the first scenario. Square and circular markers denote the initial and final positions of the players, whereas arrows indicate the direction of travel.

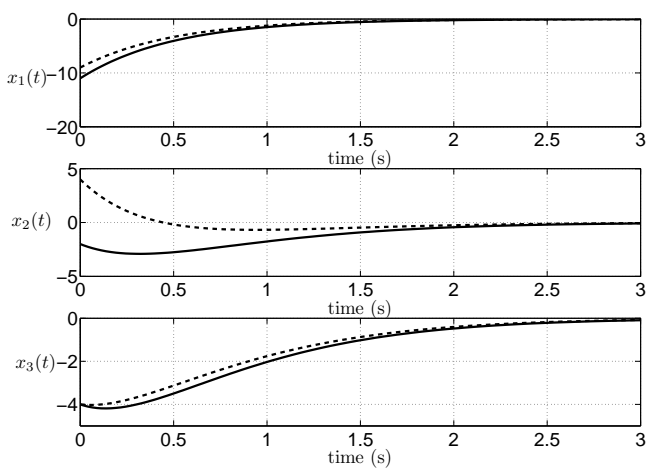

Fig. 5. Time histories of the first (solid lines) and second (dashed lines) of $x_{1}$ (top), $x_{2}$ (middle) and $x_{3}$ (bottom) corresponding to the second scenario.

and third player, respectively. The square and circular markers indicate the initial and final positions of the players and the arrows indicate the direction of travel.

\section{CONCLUSiOnS AND Future WORK}

A differential game with limited communication is considered in this paper: the communication topology is represented by a directed graph. The problem is formally defined and sufficient conditions for a solution are presented in both the general nonlinear setting and the linear-quadratic setting. A numerical example describing a multi-agent system is then introduced to illustrate the results.

Some directions for future research are as follows. It is of interest to derive weaker conditions than that in (5). This is interesting from both a theoretical and practical point-of-view: several applications which can be modeled as in the differential game framework are subject to limited communications and, in some cases, it may be difficult to satisfy the conditions presented in this paper. It is also of interest to study the impact which the communication topology has on how easily 


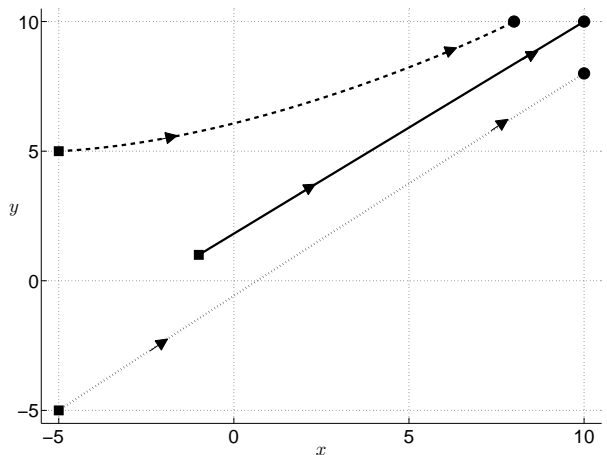

Fig. 6. Time trajectories of $p_{1}$ (solid line), $p_{2}$ (dashed line) and $p_{3}$ (dotted line) on the $x, y$-plane corresponding to the second scenario. Square and circular markers denote the initial and final positions of the players, whereas arrows indicate the direction of travel.

a solution to the PDEs (4) (or the AREs (8) in the linear-quadratic case), $i=1, \ldots, N$, can be found and whether the conditions (5) (or (9) in the linear-quadratic case), $i=1, \ldots N$ are satisfied. In the first instance, this will be done by considering specific problems and exploring the effect which the communication topology has on these problems. Finally, since closed-form solutions to the PDEs (4), $i=1, \ldots, N$, are usually not readily obtainable it is desirable to develop methods of systematically constructing approximate solutions, possibly in the spirit of the methods developed in [20] for standard differential games and in [31] for optimal control problems.

\section{REFERENCES}

[1] T. Basar and G. Olsder, Dynamic Noncooperative Game Theory. Academic Press, 1982

[2] A. W. Starr and Y. C. Ho, "Nonzero-sum differential games," Journal of Optimization Theory and Applications, vol. 3, pp. 184206, 1969.

[3] — , "Further properties of nonzero-sum differential games," Journal of Optimization Theory and Applications, vol. 3, pp. 207$219,1969$.

[4] I. N. Katz, H. Mukai, H. Schattler, and M. Zhang, "Solution of a differential game formulation of military air operations by the method of characteristics," in American Control Conference, vol. 1, 2001, pp. 168-175.

[5] E. Dockner, G. Feichtinger, and A. Mehlmann, "Noncooperative solutions for a differential game model of fishery," Journal of Economic Dynamics and Control, vol. 13, no. 1, pp. 1-20, 1989.

[6] G. Neumann and S. Schuster, "Continuous model for the rock scissors paper game between bacteriocin producing bacteria," Journal of Mathematical Biology, vol. 54, no. 6, pp. 815-846, 2007.

[7] M. L. Zeeman, "Extinction in competitive Lotka-Volterra systems," Proceedings of the American Mathematical Society, vol. 123, no. 1, pp. 87-96, 1995.

[8] M. E. Hibbing, C. Fuqua, M. R. Parsek, and S. B. Peterson, "Bacterial competition: surviving and thriving in the microbial jungle," Nature Reviews Microbiology, vol. 8, pp. 15-25, 2010.

[9] J. Hofbauer and K. Sigmund, "Evolutionary game dynamics," Bulletin of the American Mathematical Society, vol. 40, pp. 479-519, 2003.

[10] W. Saad, Z. Han, H. Poor, and T. Basar, "Game-theoretic methods for the smart grid: An overview of microgrid systems, demand-side management, and smart grid communications," Signal Processing Magazine, vol. 29, no. 5, pp. 86-105, Sept 2012.
[11] W. Weaver and P. Krein, "Game-theoretic control of small-scale power systems," IEEE Transactions on Power Delivery, vol. 24 no. 3, pp. 1560-1567, July 2009.

[12] N. Ekneligoda and W. Weaver, "Optimal transient control of microgrids using a game theoretic approach," in Energy Conversion Congress and Exposition (ECCE), Sept 2011, pp. 935-942.

[13] T. Mylvaganam and A. Astolfi, "Control of microgrids using a differential game theoretic framework," in 54th IEEE Conference on Decision and Control, 2015.

[14] T. Mylvaganam, M. Sassano, and A. Astolfi, "A constructive differential game approach to collision avoidance in multi-agent systems," in American Control Conference, 2014.

[15] T. Mylvaganam and A. Astolfi, "A differential game approach to formation control for a team of agents with one leader," in American Control Conference, 2015.

[16] R. Vinter, Optimal Control. Birkhäuser, 2000.

[17] M. Simaan and J. B. Cruz, "On the Stackelberg strategy in nonzero-sum games," Journal of Optimization Theory and Applications, vol. 11, no. 5, pp. 533-555, 1973.

[18] J. Nash, "Non-cooperative games," Annals of Mathematics, vol. 54, no. 2, pp. 286 - 295, September 1951

[19] A. Bensoussan, S. Chen, and S. P. Sethi, "The maximum principle for global solutions of stochastic stackelberg differential games," 2012

[20] T. Mylvaganam, M. Sassano, and A. Astolfi, "Constructive $\epsilon$-Nash equilibria for nonzero-sum differential games," IEEE Transactions on Automatic Control, vol. 60, no. 4, pp. 950-965, 2015.

[21] J. Baillieul and P. Antsaklis, "Control and communication challenges in networked real-time systems," Proceedings of the IEEE, vol. 95, no. 1, pp. 9-28, 2007.

[22] W. Lin, Z. Qu, and M. A. Simaan, "Nash strategies for pursuitevasion differential games involving limited observations," IEEE Transactions on Aerospace and Electronic Systems, vol. 51, no. 2, pp. 1347-1356, 2015

[23] S. Bopardikar, F. Bullo, and J. Hespanha, "On discrete-time pursuit-evasion games with sensing limitations," IEEE Transactions on Robotics, vol. 24, no. 6, pp. 1429-1439, 2008

[24] T. Mylvaganam and A. Astolfi, "Approximate solutions to a class of nonlinear stackelberg differential games," in 53rd IEEE Annual Conference on Decision and Control, 2014, pp. 420-425.

[25] M. Mesbahi and M. Egerstedt, Graph Theoretic Methods in Multiagent Networks, 1st ed. Princeton University Press, 2010.

[26] S. Martinez, J. Cortes, and F. Bullo, "Motion coordination with distributed information," IEEE Control Systems Magazine, vol. 27, no. 4, pp. 75-88, 2007.

[27] H. Tanner, A. Jadbabaie, and G. Pappas, "Flocking in fixed and switching networks," IEEE Transactions on Automatic Control, vol. 52, no. 5, pp. 863-868, 2007.

[28] R. Bellman, "The theory of dynamic programming," DTIC Document, Tech. Rep., 1954.

[29] J. Engwerda, LQ dynamic optimization and differential games. Chichester: John Wiley \& Sons, 2005.

[30] A. J. T. M. Weeren, J. M. Schumacher, and J. C. Engwerda, "Asymptotic analysis of linear feedback nash equilibria in nonzero-sum linear-quadratic differential games," Journal of Optimization Theory and Applications, vol. 101, no. 3, pp. 693-7221, 1999.

[31] M. Sassano and A. Astolfi, "Dynamic approximate solutions of the $\mathrm{HJ}$ inequality and of the HJB equation for input-affine nonlinear systems," IEEE Transactions on Automatic Control, vol. 57 pp. 2490-2503, 2012. 